| Bone and Joint Decade 2000-2010 in Nepalese Perspective

\author{
Paudyal BP' \\ 'Department of Medicine, Patan Academy of Health Sciences, Patan Hospital, Kathmandu, Nepal.
}

\begin{abstract}
The Bone and Joint Decade is a worldwide multi-disciplinary initiative targeting the care of people with musculoskeletal disorders. It focuses on improving the quality of life of these people through advances in the understanding and treatment through education, research, and prevention strategies. Though the epidemiological data in Nepal are very scanty, worldwide statistics have shown that musculoskeletal conditions represent more than half of all chronic diseases, and are the most common cause of severe long-term pain and disability.

There is acute shortage of specialist manpower that care for people with these disorders. Furthermore the teaching in musculoskeletal disorders in health institutions is very minimal. All of these problems highlight the need for expanding the teaching of musculoskeletal disorders at all levels of medical education; promotion of clinical research; improving diagnostic, treatment, and rehabilitative facilities; and implementation of preventive strategies to reduce the burden of these chronic debilitating conditions in the society.
\end{abstract}

Keywords: bone and joint decade, musculoskeletal and rheumatic diseases, Nepal

\section{BONE AND JOINT DECADE 2000-2010}

The World Health Organization (WHO) launched the Bone and Joint Decade (BJD) in January 2000 in response to the overwhelming epidemic of musculoskeletal diseases all over the world. ${ }^{1}$ This is a global, multidisciplinary initiative targeting the care of people with musculoskeletal conditions, in particular bone and joint disorders. Though this year marks the end of this decade, the importance of this initiative is equally relevant and even more useful for countries like Nepal where musculoskeletal disorders are a major cause of morbidity and majority of the health care professionals are unaware about this initiative.

The WHO/World Bank Global Burden of Disease Project showed that musculoskeletal conditions represent more than half of all chronic conditions and are the most common cause of severe long-term pain and physical disability. ${ }^{2}$ Data from individual countries also highlight the significant burden of these diseases. For example, in Australia, musculoskeletal diseases are the second most common cause of presentation to a general practioner, and the third leading cause of health system expenditure. ${ }^{1}$ In the United States, approximately

\footnotetext{
Correspondence:

Dr. Buddhi P Paudyal

Department of Medicine

Patan Academy of Health Sciences

Patan Hospital Kathmandu, Nepal.

Email: buddhipaudyal@yahoo.com

Phone: +977-1-5522278
} 
$20-30 \%$ of the general population has symptoms of arthritis or soft tissue rheumatism. ${ }^{3}$ Aging populations throughout the developed and developing world will further lead to significant increases in musculoskeletal conditions, with attendant increases in both direct and indirect costs.

With the hope of significant reduction in expected increase in joint destruction by arthritis, osteoporotic fractures, severe limb and back injuries, and other musculoskeletal disorders, the WHO set four major aims of Bone and Joint Decade campaign. ${ }^{4}$

To raise awareness about the growing burden of musculoskeletal disorders in the society;

To promote prevention of musculoskeletal disorders and empower patients through education campaigns;

To advance research on prevention, diagnosis and treatment of musculoskeletal disorders; and

To improve diagnosis and treatment of musculoskeletal disorders including diseases causing disability to musculoskeletal trauma.

Based on these objectives of Bone and Joint initiative, several countries set up their national agenda to fulfill these goals. However, no such planning and activities are known to have launched in Nepal. In this article, the author wishes to discuss the main domains of musculoskeletal disorders and their relevance in Nepal.

\section{EXTENT OF THE PROBLEM: MUSCULOSKELETAL DISEASES IN NEPAL}

The spectrum of musculoskeletal conditions in less developed countries is generally similar to that in developed countries, except that severity may be worse because of late presentation and lack of effective treatment. However, there are virtually no epidemiological data on musculoskeletal disorders in Nepal. Limited hospital-based statistics show that soft tissue rheumatism, inflammatory arthritis (rheumatoid arthritis and others), osteoarthritis, and connective tissues diseases are the common rheumatic presentations. ${ }^{5,6}$

The differences in access to treatment to these diseases result in higher disability rates in developing countries. The burden will again change largely due to increasing life expectancy in developing countries, as musculoskeletal conditions are often lifelong.

The major musculoskeletal and rheumatic problems in any community include joint diseases (osteoarthritis, rheumatoid arthritis, gout, spondyloarthritis etc), back pain, osteoporosis and other bone diseases, and trauma and injuries. Infections and congenital and developmental anomalies of the musculoskeletal system are also significant problems in developing countries.

Joint problems are common universally. Osteoarthritis is the most common joint condition, and causes significant disability. Data from various countries show the prevalence of this disorder in the range of $10-20 \%{ }^{7}$ and this is likely to rise as the age of the population advances. Osteoarthritis of knees, cervical spine and lumbar spine are common causes of outpatient visits in all hospitals and across all specialties. Rheumatoid arthritis has a worldwide prevalence of $1 \%$, though this may vary between populations. ${ }^{7}$ Many patients with this disease are likely to remain undiagnosed and inadequately treated in country like ours because of limited health-care services, lack of access to expensive treatments, and inadequate medical education. Spondyloarthritis, particularly reactive arthritis may be more common in developing countries like ours owing to the high prevalence of predisposing infections, particularly gastrointestinal and urogenital infections, though prevalence of HLA B27, a genetic predisposing factor, is still unknown. Septic arthritis, tuberculosis, and infections related to HIV/AIDS are also more common in developing countries. Back pain is a universal problem; almost everybody in his/her lifetime gets a backache. Osteoporosis and resultant fragility fractures are largely age-related due to reduced bone strength. They occur in all societies, but will increase with aging of the population. Musculoskeletal trauma and road traffic injuries are increasing precipitously. In Nepal, injuries were the significant cause of morbidity and the third leading cause of death contributing $9 \%$ of total mortality as per 1998-1999 estimates; and road traffic accidents were the $8^{\text {th }}$ position in the overall ranking. ${ }^{8}$ By the year 2010 musculoskeletal trauma are estimated to account for as much as $25 \%$ of all health care expenditures in developing nations. ${ }^{9}$ The musculoskeletal injuries can have various long term impacts: neck and back injuries result in chronic pain whereas lower limb and upper limb injuries often result in decreased mobility and loss of manual dexterity respectively. Work-related problems are another common cause of musculoskeletal pain in the society, though their frequency is not often documented in developing countries.

\section{RISK FACTORS FOR MUSCULOSKELETAL PROBLEMS}

The risk factors for the development of musculoskeletal conditions are similar to all societies, although the exposure to these risks varies in different parts of the world. Common known risk factors include obesity, lack of physical activity, poor diets (including lack of protein, calcium, and vitamin D), smoking, alcohol, injuries, repetitive work-related activity, and various diseases and infections. Many of these risk factors are becoming 
increasingly common in Nepal. Obesity, sedentary lifestyle, and injuries are particularly associated with osteoarthritis and back pain. Smoking, occupational exposures to pollutants and various infections have been shown to be associated with higher risk of developing rheumatoid arthritis. ${ }^{10}$ Smoking on the other hand, along with alcohol consumption, and vitamins and mineral deficiency (particularly calcium and vitamin D) is related with metabolic bone diseases like osteoporosis. Diseases like rickets and osteomalacia are often associated with deficiency of calcium and vitamin $D$, a common deficiency in Nepal.

\section{PREVENTIVE AND TREATMENT STRATEGIES OF MUSCULOSKELETAL DISORDERS}

Surveys in Europe and other developed countries have shown that people with musculoskeletal pain often choose to suffer the symptoms and associated disability rather than seeking active medical interventions. ${ }^{11}$ There may be various reasons for this: perception that pain should not be masked, treatments may not be effective, hazards of treatment outweighing benefits etc. Furthermore, many people think that little can be done for these problems and health-care professionals are inadequately trained about musculoskeletal conditions.

With the advancement of research in this field, there are now effective treatments for many musculoskeletal conditions, as well as many effective methods to control symptoms and maintain physical function. The spectrum of interventions includes health education, changes in lifestyle and environment, provision of dietary supplements, pharmacological agents for symptom control or disease modification, and surgery or rehabilitative interventions. ${ }^{12}$ In this regard, many European countries have recommended that people of all ages should be encouraged to follow a 'bone-andjoint healthy lifestyle' to avoid the specific risks related to musculoskeletal health. ${ }^{13}$ These recommendations are relevant for several musculoskeletal conditions and across different countries, more so for developing countries. Considering the burden of musculoskeletal diseases and their resultant functional disability, these interventions are even more important for Nepal. The bone-and-joint healthy lifestyle consists of various activities like encouragement to maintain physical fitness, maintenance of an ideal body weight, use of balanced diet containing adequate calcium and vitamin $D$, and avoidance of smoking and excessive alcohol consumption. In the society, the possible intervention programs include promotion of accident prevention strategies, health promotion at work place and during sports activities, and greater public awareness on early diagnosis and management of musculoskeletal problems. $^{13}$
Taking into account the chronic debilitating nature, high functional disability, and overwhelming socioeconomic loss due to musculoskeletal diseases, time has come to focus on prevention and early treatment strategies. This is even more important for resource poor and developing countries like Nepal.

\section{EDUCATION ON MUSCULOSKELETAL DISORDERS IN MEDICAL INSTITUTIONS}

Patients with complaints about bones and joints are often ignored and their problems underestimated by doctors. This is because medical teaching in musculoskeletal disorders in all parts of the world is currently brief and not directly relevant to the knowledge and skills commonly required for the management of these conditions in an outpatient setting. ${ }^{14} \mathrm{~A}$ survey on the undergraduate curriculum in the Asia-Pacific region showed that musculoskeletal teaching represented approximately $2 \%$ of the total contact hours in the medical schools. ${ }^{15}$

Musculoskeletal complaints are the second most common reason for consultation, exceeded only by disorders of the respiratory system. To cope with this heavy burden of musculoskeletal problems, the World Health Organization (WHO) has recommended at least one rheumatologist for every 100,000 population. However, the real scenario is far from this goal: in the early 1990s, excluding Japan, New Zealand and Australia, there were probably less than 100 fully trained rheumatologists for the rest of the Asia Pacific region with a population of nearly three billion people. 15 In Nepal, though the number of general physicians and orthopedic surgeons is gradually increasing to meet the growing burden of musculoskeletal diseases, there is acute dearth of specialist manpower in the field of rheumatic diseases. For example, in 2009, there were only two rheumatologists (one currently out of country) and few more rheumatology practioners for a population of nearly 30 million in the whole country.

This highlights the need for greater involvement of musculoskeletal conditions in undergraduate and postgraduate levels of training. Strategies have to be implemented to focus on education of all health professionals, beginning with the training to middle level health workers, and undergraduate medical curriculum. Creation of training opportunities for specialist manpower, development of faculties in central hospitals, and establishment of good basic science services like immunology and laboratory medicine are essential for the promotion of musculoskeletal medicine in the country. 


\section{RESEARCH IN MUSCULOSKELETAL/ RHEUMATIC DISEASES}

Rheumatology is in infancy of its development in Nepal, both in terms of clinical facilities and research. There is gross paucity of data on epidemiology and impact of various rheumatic diseases, mainly due to lack of resources. For example, we do not have data on pattern, prevalence, associated risk factors and outcome of common rheumatic diseases like rheumatoid arthritis, spondyloarthritis, systemic lupus erythematosus, osteoarthritis, gout, and various others, which is essential for planning and allocating resources for treatment and preventive programs. Thus good quality clinical research is important not only to better understand these diseases in our own setting but also to plan effective control measures in the future.

\section{SUMMARY}

We are currently at the exit of the Bone and Joint Decade 2000-2010, an initiative to curb the growing but underrecognized burden of the rheumatic and musculoskeletal diseases all over the World. Combined, these diseases are the cause of significant physical disability and premature mortality, and are a major burden to the society with high health services spending and labor loss. Expanding the teaching of musculoskeletal disorders in all levels of medical training, promotion of basic and clinical research to understand the epidemiology and impact of these diseases, improving diagnostic, treatment, and rehabilitative facilities, and effective implementation of preventive strategies are some of the key steps that help to reduce the burden of these chronic debilitating conditions in the society.

\section{REFERENCES}

1. Brooks PM, Hart JAL. The Bone and Joint Decade: 2000-2010. Med J Aust. 2000; 172: 307-8.

2. Murray JL, Lopez AD, eds. The global burden of disease: a comprehensive assessment of mortality and disability from diseases, injuries and risk factors in 1990 and projected to 2020. Cambridge, Mass: Harvard University Press;1996.

3. Lawrence RC, Helmick CG, Arnett FC, et al. Estimate of the prevalence of arthritis and selected musculoskeletal disorders in the United States. Arthritis Rheum 1998; 41: 778- 99

4. Hazes JM, Woolf AD. The bone and joint decade 2000-2010. J Rheumatol 2000; 27: 1-3

5. Das RN, Paudel R. Spectrum of rheumatological disorders: an experience of 337 cases in a tertiary care hospital in Pokhara valley, Nepal. APLAR Journal of Rheumatology. 2006; 9:24856

6. Paudyal B. Pattern of Rheumatic Disease Among Patients Attending Rheumatic Disease Clinic at Patan Hospital. Souvenir of the $24^{\text {th }}$ All Nepal Medical Conference of the Nepal Medical Association. 2009; p. 56

7. Woolf AD, Pfleger B. Burden of musculoskeletal conditions. Bulletin of the World Health Organization 2003;81:646-55

8. Jha N. Road Traffic Accidents: An Emerging Problem in Nepal. J Nep Med Assoc 2005; 44: 156-9

9. Goyal S. Bone and Joint Decade (BJD) 2000-2010. Indian Journal of Orthopaedics 2006; 40: 200-1

10. Harris ED, Schur PH. Epidemiology, risk factors for, and possible causes of rheumatoid arthritis. UpToDate, September 2009

11. Woolf AD, Zeidler H, Haglund U et al. Musculoskeletal pain in Europe: its impact and a comparison of population and medical perceptions of treatment in eight European countries. Annals of the Rheumatic Diseases 2004; 63: 342-7

12. Woolf AD, Brooks P, Akesson K, Mody GM. Prevention of Musculoskeletal conditions in the developing world. Best Practice \& Research Clinical Rheumatology 2008; 22: 759-72

13. European action towards better musculoskeletal health. Lund, Sweden: Bone and Joint Decade. ISBN 91-975284-0-4; 2004. Available from: URL:http://europa.eu.int/cbmmhealth/ ph_projects/2000/promotion/fp_promotion_2000_exs_15_ en.pdf

14. Akersson K, Dreinhofer KE, \& Woolf AD. Improved education in musculoskeletal conditions is necessary for all doctors. Bulletin of the World Health Organization 2003; 81: 677-82

15. Mody GM, Handa R. Preface to the Musculoskeletal Conditions in the Developing World. Best Practice \& Research Clinical Rheumatology 2008; 22:579-8 